\title{
Microtubule-Associated Proteins 1A/1B Light Chain 3-II
}

National Cancer Institute

\section{Source}

National Cancer Institute. Microtubule-Associated Proteins 1A/1B Light Chain 3-II. NCI

Thesaurus. Code C105644.

Microtubule-associated proteins 1A/1B light chain 3-II proteins (LC3-II) are products of the autophagy-dependent processing of microtubule-associated proteins $1 \mathrm{~A} / 1 \mathrm{~B}$ light chain 3 family proteins (LC3). During autophagy, LC3 proteins are cleaved by cysteine protease AT G4B to form LC3-I intermediates which are located in the cytoplasm. As autophagy progresses, LC3-I proteins interact with both ubiquitin-like modifier-activating enzyme ATG7 and ubiquitin-like-conjug ating enzyme ATG3. These interactions result in further proteolysis and the conjug ation of a phospholipid anchor to form the membrane localized LC3-II. 